\title{
Research on Opportunities of the Internet Industry Under the COVID-19 Epidemic
}

\author{
Yan Fang, Guihua Guo, Ying Hu, Lijiao Zhuang
}

\author{
School of Business, Jiangxi Normal University, Nanchang 330022, China \\ *Corresponding author. Email: 1174672453@qq.com
}

\begin{abstract}
During the epidemic, problems such as lack of materials, lack of funds, and lack of labor have emerged one after another. The emergence of the new Internet + model uses its unique advantages of digital information aggregation to deploy medical supplies, expand financing channels, and help most companies to resume work remotely, alleviating the epidemic impact on China. Although it is the focus of the development of the times, there are still certain problems in its progress. It needs to continuously improve its own development system to escort the development of the industry.
\end{abstract}

Keywords: new crown epidemic, industrial internet, SMEs

\section{INTRODUCTION}

In 2020, the sudden outbreak of COVID-19pneumonia has caused huge impacts and losses throughout the world. Due to its "person-to-person transmission" characteristics, the original plans of various industries were disrupted one after another. As a major strategic deployment of China in recent years, the Internet has been fully and deeply advanced, especially during the epidemic period, showing the important role of digitalization in resuming work and production and facing the impact of uncertainty, making more people aware of its preciousness. A large number of scholars have made important contributions to it during the epidemic [1-2]. However, the description of the emergence of its opportunities is still lacking in a certain degree of systematic way of approaching it.

This article systematically explains the development process of the Internet during the epidemic based on the difficulties during the epidemic period, and the new development model as well as the development opportunities of the Internet.

\section{VARIOUS NEW PROBLEMS DURING THE COVID-19 EPIDEMIC}

The Spring Festival without fireworks, firecrackers, and New Year's greetings is just the beginning of the arrival of the Rat Year at the beginning of the year. Unexpectedly, more and more difficult things did follow.

Lack of medical supplies: The arrival of the epidemic has made scarce medical supplies even more valuable. Due to the insufficient production capacity of relevant protective medical products, and the continuous road closures in various places, the roads for the transportation of medical supplies have become more difficult; moreover, the roads for information dissemination are also blocked, has caused people in need cannot be rescued in time. Under greater threat, the efficiency of social linkage has declined.

Lack of trading channels: The daily red figures are constantly climbing, which seems to be a death race. Wuhan, where the epidemic first appeared, is even more difficult. Road closures began across the country, from the closure of highways in various provinces, cities, and counties to the closure of roads in various communities and villages. The closure of roads makes it difficult for merchants such as vegetable farmers, fruit farmers, and supermarkets to sell their products. Consumers who are isolated from home and obstructed the purchase of daily necessities have greatly affected their lives.

Lack of labor: As a large manufacturing country, laborintensive enterprises play a vital role in my country's national economy. Today, the epidemic occurs around the Spring Festival, the whole country is isolated, and many industrial activities are stagnant [3-4]. It is difficult to communicate information between employees seeking employment and the recruitment of employees of enterprises that have resumed work, resulting in conflicts between supply and demand, such as difficulties in recruitment for enterprises and difficulties in finding jobs for workers and affecting the stability of the job market. Lack of funds: It is difficult to start work and the resumption rate is low, but factory rents, wages, interest, etc. must continued to be paid, leading to increased costs for the company; besides that, the sharp decline in the business volume of the company and the substantial increase in production costs has also caused cash flow problems [5-6]. Although financing is also a way to solve capital problems, today's financing costs are high and there are few available channels. At the same time, high financing costs and interest make it difficult for companies.

The sudden epidemic has brought us many new tests, such as channel blockage, isolation of personnel, death threats... However, we are all fearless, and learning to grow in adversity is the driving force for our progress. 


\section{INTERNET + NEW MODELS}

The arrival of the epidemic has caused many Chinese manufacturing companies facing the dilemma of forced suspension of production, and the development of the global manufacturing industry will not stop due to the suspension of production in some areas. The Industrial Internet has shown its own unique advantages and has become an emergency response. An important tool for improving efficiency, giving full play to the advantages of information aggregation, online operations, remote collaboration, remote services, etc., can effectively connect all elements of industrial production, the entire industry chains, and the entire value chains, and realize the production and dispatch of key materials in a short time, The overall optimization of distribution, to help enterprises realize the advantages of industrial Internet technology in an efficient, convenient, online, and collaborative working mode, and to provide solutions to many problems that occurred during the epidemic.

Three of the most important Internet + new models have constructed new models of medical care, resumption of work and resumption of school. Internet + medical care, to give full play to the advantages of data aggregation, resource scheduling, data analysis, etc., to improve the efficiency of emergency response during the epidemic. Internet medical service platforms such as Tencent Medical Dictionary, WeDoctor, Good Doctor Online, Penguin Almond, and Medical Federation have recruited 10,000 licensed doctors from all over the country to continuously expand online free consultations for the COVID-19 epidemic to greatly avoid cross-infection caused by diagnostic errors and alleviate pressure from medical staff [7]. Internet + education has overcome the delayed in opening of schools, timely online courses, cloud classrooms and other Internet tools, have ensured that "classes are not suspended". Educational departments in all regions have postponed the opening hours of universities, middle schools, elementary schools and kindergartens. It is necessary to take full advantage of the infrastructure of online education platforms and the high penetration rate of smartphones to encourage teachers and students to study online; Internet + office: with theCOVID-19, the prevention and control of this pneumonia epidemic continues to be upgraded, and the online service support of the Internet has expanded from personal services to government services and corporate services. It also provides enterprise remote office support to reduce the frequency of front-line personnel going to and from the factory. The remote collaboration function based on the industrial Internet helps corporate employees to achieve home office and online operations, and solve the problem of labor shortage and difficulty in resuming work.

In addition, the emergence of Internet+sales and Internet+finance models have not only facilitated the precise connection between customers and upstream suppliers, but also connected financial institutions and manufacturing companies to resolve capital problems, and relieved the financial pressure of the epidemic. .

In the face of adversity, the Internet continues to accelerate the development of new technologies, new business forms and new models, providing new development opportunities for China's economy, and driving a substantial increase in the application demand for new generation information technology. Industrial Internet has become one of the new highlights of China's economy, and nurturing huge new development momentum while ushering in new development opportunities for the industry, it also made a huge contribution to the stability of social life in China during the epidemic.

\section{OPPORTUNITIES FOR THE DEVELOPMENT OF THE INTERNET INDUSTRY}

\subsection{Frequent New Deals Drive the Industry to Accelerate Development.}

The National Development and Reform Commission, the Central Cyberspace Administration of China, the Ministry of Education and other 13 departments jointly issued the "Opinions on Supporting the Healthy Development of New Business Forms and New Models, Activating the Consumer Market and Driving Employment Expansion", which proposes to "consolidate online and offline new business forms and new models as economic tools. An important breakthrough for transformation and promotion of reform and innovation," and said that "digital innovation can effectively play a leading role in improving the quality and efficiency of the real economy, and promote 'Internet +', big data, and platform economy to a new stage."

\subsection{Increase Fiscal and Taxation Support for Key Guarantee Enterprises}

Set up a special re-loan of 300 billion yuan to support financial institutions to provide loan concessions to key enterprises during the epidemic. In order to further promote the support of local finances, the central government has even introduced the actual loan interest rate $50 \%$ subsidies.

For enterprises that expand production capacity and purchase production equipment, allowing a one-off deduction of relevant taxes.

The importation of essentialmaterials for epidemic prevention and control have been exempted from valueadded tax. 


\section{DEVELOPMENT SUGGESTIONS AND STRATEGIES FOR THE INTERNET INDUSTRY}

\subsection{Suggestion}

The emergence of the Internet + new model has certainly solved many problems that existed during the epidemic. However, while maintaining the stable development of society, we must also focus on cooperating with the government to solve industry problems and improve the industry development system in order to accelerate our own development.

Strengthen self-discipline in the Internet industry. First of all, companies in the Internet industry and the financial industry must strengthen their alliances to jointly resist illegal disclosure of information. At the same time, in order to realize information sharing, a platform for regular information disclosure needs to be established; on the basis of reaching a consensus on cooperation, it is still necessary to establish supervision of Organization. In addition to inter-industry cooperation, the correct guidance from government departments is also essential.

Promote the construction of a credit information system. In the era of information sharing, enhancing mutual trust is the key to building a bridge for cooperation between the financial and Internet industries. Therefore, the credit reporting system for both are needed to be put on the agenda. In view of the characteristics of these two industries in China, we should construct the system from the following two aspects: Firstly, expand the scope of China's existing credit investigation system and break the current lack of credit records for small and micro enterprises and individuals. Secondly is to increase penalties for those who violate the contract, and at the same time give the victims certain compensation.

Improve related regulatory systems. In terms of legal supervision, relevant government departments need to introduce relevant rules and regulations to fill the existing legal gaps, make behaviors in accordance with laws and promote fair resolution of problems, and escort the healthy development of the IT industry. On the other hand, in terms of institutional supervision, it is necessary to comprehensively supervise the financing models of the two industries, namely, Tongjin and the Internet.

\subsection{Development Strategy}

In the short term, Internet companies can still play a greater role in the epidemic prevention and control. The epidemic prevention and control has reached a critical stage, and community prevention and control is the focus. The prevention and control measures taken in various places are becoming more and more stringent. A large amount of manpower and material resources have been invested in the grassroots through grid management, and closed management of the community has been adopted.
These prevention and control measures are working, but most of these measures still remain in the pre-Internet era, such as man-to-man, dragnet investigation, manual form filling, and crowd tactics. The tasks of grassroots civil servants are heavy. It is necessary to actively strengthen communication and cooperation with relevant Internet companies, strengthen information technology support for community prevention and control, and rapidly develop epidemic management tools for community prevention and control scenarios. We should open up and open up more inter-departmental and inter-regional data, break information silos, take advantage of Internet companies, adopt more digital, networked, and intelligent means, develop and use more Internet big data tools and digital means, and help improve collaborative defense Control mechanism, rationally allocate resources, and improve the efficiency of prevention and control.

In the medium and long term, digital transformation should be further promoted and the major epidemic risk prevention and control system should be improved. Further play the role of digital technologies such as 5G, big data, artificial intelligence, and the Internet of Things in epidemic prevention, monitoring, and response, improve the public health monitoring system, and build epidemic risk assessment tools and frameworks. Strengthen the construction of a digital platform for the public health system, improve the efficiency of medical facilities and resource matching, strengthen hierarchical diagnosis and treatment and upper and lower linkages, and improve the ability of medical institutions to serve large-scale populations. While continuing to do a good job in scientific prevention and control, promote the in-depth integration and development of the Internet and the real economy, use the Internet platform to do a good job in matching supply chain, human resources and other information and resources, and orderly resume normal production.

\section{CONCLUSION}

The contactless "Internet cloud service" during the epidemic provides a guarantee for the orderly progress of social life, and the development of the Internet is not restricted by the end of the epidemic. It is an important trend in future development. Bring more convenience to our lives. In the process of continuous development, relevant government departments should give more support to the Internet industry, and at the same time, they should also focus on their own improvement and upgrading. 


\section{REFERENCES}

[1] Xuesong Zhou: "The Epidemic Forces China's Economic Development" [N], China Economic Times, 2020.

[2] Yiliang Chen: "Enterprises under the epidemic situation, travel, hotel and catering industry encounters a cold wave, Internet industry ushered in opportunities, listed companies switch to epidemic prevention materials" [J]. China Economic Weekly.

[3] Xuechen Wen: The epidemic has spawned demand for Internet medical services. The industry will accelerate growth soon $[\mathrm{N}]$. China Industry News.

[4] CCID Consulting: Post-epidemic era: How will the digitalization of Chinese manufacturing develop? [N]. China Computer News.

[5] Xinyuan Zhang: Wang Mingji. Research on the Financing of Small and Medium-sized Enterprises under the Internet Finance Model [J]. Cooperative Economy and Technology.

[6] Hongni Jian. "Cloud Economy" Promotes Hubei's Recovery [J]. Xiaokang, 2020(24): 61-63.

[7] Haiyi Wu. "Internet + medical insurance" makes it easier for the masses to seek medical advice [N]. Zhuhai Special Zone News, 2020-08-28 (004). 